\title{
Dependence of Judgments in Analytic Hierarchy Process: A Statistical Aspect
}

\author{
Prasanta Basak \\ Penn State Altoona \\ Altoona, PA
}

\author{
Indrani Basak \\ Penn State Altoona \\ Altoona, PA
}

\begin{abstract}
In Analytic Hierarchy Process, the judgment on a pair of treatments or objects may affect the judgment on other pair of treatments especially when these two pairs have a treatment in common. This dependence could be established by using a model developed by Bahadur [1]. In this model, we test the presence of order effect of presentation within a pair of objects. Any two pairs of alternatives are tested for the correlation between them. It is also tested whether a certain number of pairs have the same degree of correlations. Priority vector, defined in terms of the order effect parameters, is estimated and the hypothesis of equal priority of objects is tested.
\end{abstract}

Keywords: correlation, dependence, iterative scheme, order effect, priority vector.

\section{Introduction}

Most of the time judgment on a pair of treatments or objects affect the judgments on other pairs of treatments, especially when these pairs have a treatment in common. Not much work has been done in this area, which recognizes the dependence of the judgments on these pairs of treatments. De Jong [7] estimates the priority vector by the log least squares method, which incorporates this type of dependence. But the model used by him does not accommodate the order effects or correlation aspects.

We use a model developed by Bahadur [1] to establish the dependence of judgments between pairs of treatments. Several authors have previously studied within-pair effect (see [2], [3], [4]), and multivariate comparison experiments (see [5], [6]). In fact, Davidson and Bradley [5] used the model of Bahadur [1] to allow the dependence of judgments among several criteria. There they assumed that the judgments on the pairs of alternatives are independent, while those on several criteria for a fixed pair are dependent. In that paper, an iterative scheme has been given to estimate the priority weights. However, its convergence to the maximum likelihood estimates is not shown. We feel that it is more worthwhile to recognize the dependence of the judgments on the different pairs involving a common alternative.

In section 2, we develop the model involving the parameters of order effect of presentations and correlations between the judgments. Order effects are estimated by the maximum likelihood procedure. We give an iterative scheme for the solution of the likelihood equations. It is also shown that the proposed iterative scheme converges to the maximum likelihood estimates. Section 3 deals with the likelihood ratio test criterion to test whether the order of presentation of the objects to judges plays a significant role. The important issue of correlation of judgments between different pairs is addressed in section 4. In section 5 , the priority vector of the objects is estimated and the equality of these weights is also tested in that section. 


\section{The Model}

Let $O_{1}, O_{2}, \cdots, O_{m}$ be $m$ objects which are to be compared pairwise with respect to certain qualitative characteristic by $n$ judges. Consider the vector $\mathbf{X}=\left(X_{12}, X_{13}, X_{14}, \cdots, X_{m-1, m}, X_{21}, X_{31}, \cdots, X_{m, m-1}\right)^{\prime}$, where,

$$
X_{i j}= \begin{cases}1 & \text { w.p. } \theta_{i j} \text { if the first item in }\left(O_{i}, O_{j}\right) \text { is preferred, } \\ 0 & \text { w.p. } \phi_{i j}=1-\theta_{i j} \text { if the first item in }\left(O_{i}, O_{j}\right) \text { is not preferred. }\end{cases}
$$

Set

$$
Z_{i j}=\frac{X_{i j}-\theta_{i j}}{\sqrt{\theta_{i j} \phi_{i j}}}
$$

and define the correlation coefficient between $Z_{i j}$ and $Z_{k l}$

$$
\rho_{i j, k l}=E\left(Z_{i j} Z_{k l}\right),
$$

Then, the probability density function of $\mathbf{X}$ is given by

$$
p(\mathbf{x})=P(\mathbf{X}=\mathbf{x})=p_{1}(\mathbf{x}) h(\mathbf{x}),
$$

where,

$$
\begin{aligned}
p_{1}(\mathbf{x}) & =\prod_{i \neq j} \theta_{i j}^{x_{i j}} \phi_{i j}^{1-x_{i j}} \\
h(x) & =1+\sum_{i j<k l} u(i j, k l) \rho_{i j, k l} Z_{i j} Z_{k l}
\end{aligned}
$$

with

$$
u(i j, k l)= \begin{cases}1 & \text { if }(\mathrm{i}=\mathrm{k} \text { or } \mathrm{i}=\mathrm{l}) \text { or }(\mathrm{j}=\mathrm{k} \text { or } \mathrm{j}=\mathrm{l}), \\ 0 & \text { otherwise. }\end{cases}
$$

Here, $i j<k l$ means that the position of $X_{i j}$ is before the position of $X_{k l}$ in the vector $\mathbf{X}$. In general, the above $p(\mathbf{x})$ need not be a probability density function. Bahadur [1] gives a necessary and sufficient condition for $p(\mathbf{x})$ to be a probability density function. In fact, Bahadur showed that if

$$
\lambda_{\min }=1-\frac{2}{\Sigma \beta_{i j}}, \text { where } \beta_{i j}=\max \left\{\frac{\theta_{i j}}{\phi_{i j}}, \frac{\phi_{i j}}{\theta_{i j}}\right\},
$$

where $\lambda_{\min }$ is the smallest eigenvalue of the correlation matrix $R=\left(\left(\rho_{i j, k l}\right)\right)$, then it defines a probability density function. We assume that (2.3) is a probability density function. Small values of $\rho_{i j, k l} \mathrm{~s}$ in absolute values would make (2.3) a probability density function. Observe that,

$$
Z_{i j} Z_{k l}=\delta\left(X_{i j}, X_{k l}\right)\left(\frac{\phi_{i j}}{\theta_{i j}}\right)^{\delta\left(X_{i j}, 1\right) / 2}\left(\frac{\phi_{k l}}{\theta_{k l}}\right)^{\delta\left(X_{k l}, 1\right) / 2}
$$

where, $\delta(.,)=$. \pm 1 , sign being positive if the arguments agree and negative otherwise.

Denote the elements of the vector $\mathbf{X}$ and corresponding $\theta$ and $\phi$ by $Y_{i}, \theta_{i}$ and $\phi_{i}$ respectively ; $i=$ $1,2, \cdots, M$, where, $M=m(m-1)$. In the light of these notations, equations (2.1), (2.2), and 2.4 reduce respectively to 


$$
\begin{aligned}
Z_{i} & =\frac{Y_{i}-\theta_{i}}{\sqrt{\theta_{i} \phi_{i}}} \\
\rho_{i j} & =E\left(Z_{i} Z_{j}\right), \\
Z_{i} Z_{j} & =\delta\left(Y_{i}, Y_{j}\right)\left(\frac{\phi_{i}}{\theta_{i}}\right)^{\delta\left(Y_{i}, 1\right) / 2}\left(\frac{\phi_{j}}{\theta_{j}}\right)^{\delta\left(Y_{j}, 1\right) / 2} .
\end{aligned}
$$

Then (2.3) can be written in terms of $\mathbf{Y}=\left(Y_{1}, Y_{2}, \cdots, Y_{M}\right)^{\prime}$ as follows.

$$
p(\mathbf{y})=P(\mathbf{Y}=\mathbf{y})=p_{1}(\mathbf{y}) h(\mathbf{y}),
$$

where,

$$
\begin{aligned}
p_{1}(\mathbf{y}) & =\prod_{i=1}^{M} \theta_{i}^{y_{i}} \phi_{i}^{1-y_{i}}, \\
h(y) & =1+\sum_{i<j} u(i, j) \rho_{i j} Z_{i} Z_{j} .
\end{aligned}
$$

Each response consists of a vector of preferences $\mathbf{y}=\left(y_{1}, y_{2}, \cdots, y_{M}\right)^{\prime}$, where component $y_{i}$ indicates which treatment in the $i$ th pair is preferred, $y_{i}=0,1 ; i=1,2, \cdots, M$. Now let $n(\mathbf{y})$ be the number of times the preference vector $\mathbf{y}$ occurs among the $n$ responses. The logarithm of the likelihood function is then given by

$$
\log L=\sum_{\mathbf{y}} n(\mathbf{y}) \log p(\mathbf{y}),
$$

where $p(y)$ is defined in (2.5) or equivalently,

$$
\log L=\sum_{i=1}^{M}\left[s_{i} \log \theta_{i}+\left(n-s_{i}\right) \log \left(1-\theta_{i}\right)\right]+\sum_{\mathbf{y}} n(\mathbf{y}) \log h(\mathbf{y}),
$$

$s_{i}=\Sigma n(\mathbf{y}) y_{i}, i=1,2, \cdots, M$ and the second sum being over all the possible $2^{M}$ values of $\mathbf{y}$ representing the preference responses. Taking derivative of (2.6) with respect to $\rho_{i j} ; i<j$ (with $u(i, j)=1$ ), $i, j=$ $1,2, \cdots, M$, and with respect to $\theta_{i} ; i=1,2, \cdots, M$, we obtain respectively,

$$
\begin{aligned}
\frac{\partial \log L}{\partial \rho_{i j}} & =\sum_{y} \frac{n(\mathbf{y})}{h(\mathbf{y})} \frac{\partial h(\mathbf{y})}{\partial \rho_{i j}}, \\
\frac{\partial \log L}{\partial \theta_{i}} & =\frac{s_{i}}{\theta_{i}}-\frac{n-s_{i}}{\phi_{i}}+\sum_{y} \frac{n(\mathbf{y})}{\partial h(\mathbf{y})} \frac{\partial}{\partial \theta_{i}},
\end{aligned}
$$

where,

$$
\begin{aligned}
\frac{\partial h(\mathbf{y})}{\delta \rho_{i j}} & =u(i, j) \partial\left(y_{i}, y_{j}\right)\left(\frac{\phi_{i}}{\theta_{i}}\right)^{\partial\left(y_{i}, 1\right) / 2}\left(\frac{\phi_{j}}{\theta_{j}}\right)^{\partial\left(y_{j}, 1\right) / 2} ; i=1,2, \cdots, M, \\
\frac{\partial h(\mathbf{y})}{\partial \theta_{i}} & =-\frac{1}{2 \theta_{i} \phi_{i}}\left(\frac{\phi_{i}}{\theta_{i}}\right)^{\delta\left(y_{i}, 1\right) / 2} \sum_{j \neq i} u(i, j) \delta\left(y_{j}, 1\right) \rho_{i j}\left(\frac{\phi_{j}}{\theta_{j}}\right)^{\delta\left(y_{j}, 1\right) / 2} .
\end{aligned}
$$


The maximum likelihood estimates $r=\left\{r_{i j} ; i, j=1,2, \cdots, M\right\}$ of the correlation parameters $\rho=\left\{\rho_{i j} ; i, j=\right.$ $1,2, \cdots, M\}$ and $q=\left\{q_{i} ; i=1,2, \cdots, M\right\}$ of the preference probabilities $\theta=\left\{\theta_{i}, i=1,2, \cdots, M\right\}$ are obtained by solving the following set of likelihood equations (with $u(i, j)=1$ ):

$$
\begin{aligned}
& \left.\frac{\partial \log L}{\partial \rho_{i j}}\right|_{\rho=r, \theta=q}=0 ; i, j=1,2, \cdots, M, \\
& \left.\frac{\delta \log L}{\delta \theta_{i}}\right|_{\rho=r, \theta=q}=0 ; i=1,2, \cdots, M .
\end{aligned}
$$

In the special case of two treatments $(m=2)$, the likelihood equations (2.7) and (2.8) can be solved explicitly to give

$$
\begin{aligned}
q_{i} & =\frac{s_{i}}{n} ; i=1,2 \\
r & =\frac{n(0,0) n(1,1)-n(0,1) n(1,0)}{\sqrt{s_{1} s_{2}\left(n-s_{1}\right)\left(n-s_{2}\right)}} .
\end{aligned}
$$

For $m>2$, explicit solutions are not possible and equations are solved iteratively for the maximum likelihood estimates. We propose the following iterative scheme to obtain solutions to the set of likelihood equations (2.7) and (2.8). The iterations are indexed by $k, k=1,2, \cdots$, since one revised value of each $q_{i}$ and $r_{i j}$ is obtained for each value of $k$. Successive values of $q$ will be subindexed by $t$ with only one $q_{i}$ being revised for each value of $t$. Details for $k$ th iteration follow in two parts.

(I) A new estimate $q$ of $\theta$ is generated cyclically through change of one element of $\theta$ at a time. The $(t+1)$ st stage value $q^{(t+1)}$ is obtained from the $t$ th stage value through replacement of the element $q_{i}^{(t)}$ only for which $t=(k-1) M+i-1, t=(k-1) M, \cdots, t=k M-1$. Let

$$
\begin{aligned}
C_{i}(\mathbf{y}) & =\sum_{j \neq i} u(i, j) \delta\left(y_{j}, 1\right) r_{i j}\left(\frac{p_{j}}{q_{j}}\right)^{\delta\left(y_{j}, 1\right) / 2}, \\
B_{i} & =\sum_{y} \frac{n(\mathbf{y})}{h(\mathbf{y})} \frac{\partial h(\mathbf{y})}{\partial q_{i}}=-\sum_{y} \frac{n(\mathbf{y}) C_{i}(\mathbf{y})}{2 h(\mathbf{y}) p_{i} q_{i}}\left(\frac{p_{i}}{q_{i}}\right)^{\delta\left(y_{i}, 1\right) / 2} .
\end{aligned}
$$

The iterative equation is defined for the two cases as follows.

Case 1: $B_{i}<0$.

$$
q_{i}^{(t+1)}=\left[1+\frac{p_{i}}{q_{i}} \sqrt[4]{\frac{q_{i}\left(n-B_{i} p_{i}\right)}{s_{i}}}\right]^{-1},
$$

where, the right hand side values are $t$ th stage values.

Case 2: $B_{i}>0$.

$$
q_{i}^{(t+1)}=\left[1+\frac{p_{i}}{q_{i}} \sqrt[4]{\frac{n q_{i}}{s_{i}+B_{i} p_{i} q_{i}}}\right]^{-1}
$$

where, the right hand side values are $t$-th stage values.

(II) The system of equations $\left.\frac{\partial \log L}{\partial r_{i j}}\right|_{k}=0 ; i<j, i=1,2, \cdots, M$ is solved by the IMSL routine ZXMWD, subject to the condition that the function $h(\mathbf{y})$ be kept positive to give $r^{(k)}$ with each $r_{i j} \in[-1,1]$. Then $r^{(k)}$ represents the solution to the system of equations for iteration $k$. Note that $\frac{\partial^{2} \log L}{\partial r_{i j}^{2}}$ is always negative indicating that the likelihood is a convex function of $r_{i j}$. 
Here, $\left.\frac{\partial \log L}{\partial r_{i j}}\right|_{k}$ denotes the value $\frac{\partial \log L}{\partial r_{i j}}$ obtained when $q^{(k m)}$ is substituted for $q$. The initial estimate $q_{i}^{(0)}$ is obtained from marginal frequencies $s_{i}, i=1,2, \cdots, M$.

We will now show that the solutions from the iterative schemes (2.9) and (2.10) converge to the maximum likelihood estimates.

Case I. $B_{i}<0$.

In this case, we can shown that $\frac{\phi_{i}^{3}}{\theta_{i}^{3}} B_{i}$ is an increasing function of $\theta_{i}$ (proved in Appendix- Lemma 2). Now,

$$
\begin{aligned}
\left.\frac{\partial \log L}{\partial \theta_{i}}\right|_{t} & =\left.\frac{\phi_{i}^{4}}{\theta_{i}^{4}}\left[\frac{\theta_{i}^{3} s_{i}}{\phi_{i}^{5}}-\frac{n \theta_{i}^{4}}{\phi_{i}^{5}}+\frac{B_{i} \theta_{i}^{4}}{\phi_{i}^{4}}\right]\right|_{t} \\
& =\left.\frac{\phi_{i}^{4}}{\theta_{i}^{4}}\left[\frac{\theta_{i}^{3} s_{i}}{\phi_{i}^{5}}-\frac{\theta_{i}^{4}}{\phi_{i}^{4}}\left(\frac{n_{i}}{\phi_{i}}-B_{i}\right)\right]\right|_{t} \\
& =\frac{\phi_{i}^{4}}{\theta_{i}^{4}}\left(\frac{n}{\phi_{i}}-B_{i}\right)\left[\left(\frac{\theta_{i}^{4}}{\phi_{i}^{4}}\right)^{(t+1)}-\left(\frac{\theta_{i}^{4}}{\phi_{i}^{4}}\right)^{(t)}\right], \text { from }(2.9),
\end{aligned}
$$

and since $\frac{\theta_{i}}{\phi_{i}}$ is an increasing function of $\theta_{i},\left.\frac{\partial \log L}{\partial \theta_{i}}\right|_{t}$ is of same sign as $\Delta \theta_{i}=\theta_{i}^{(t+1)}-\theta_{i}^{(t)}$. Again,

$$
\begin{aligned}
\left.\frac{\partial \log L}{\partial \theta_{i}}\right|_{t+1} & =\frac{\phi_{i}^{4}}{\theta_{i}^{4}}\left(\frac{n}{\phi_{i}}-B_{i}\right)\left[\left(\frac{\theta_{i}^{3} s_{i} / \phi_{i}^{4}}{n-B_{i} \phi_{i}}\right)^{(t+1)}-\left(\frac{\theta_{i}^{4}}{\phi_{i}^{4}}\right)^{(t+1)}\right] \\
& =\frac{\phi_{i}^{4}}{\theta_{i}^{4}}\left(\frac{n}{\phi_{i}}-B_{i}\right)\left[\left(\frac{\theta_{i}^{3} s_{i} / \phi_{i}^{4}}{n-B_{i} \phi_{i}}\right)^{(t+1)}-\left(\frac{\theta_{i}^{3} s_{i} / \phi_{i}^{4}}{n-B_{i} \phi_{i}}\right)^{(t)}\right]
\end{aligned}
$$

from (2.9), which has the same sign as $\Delta \theta_{i}$ since

$$
\frac{\theta_{i}^{3} s_{i} / \phi_{i}^{4}}{n-B_{i} \phi_{i}}=\frac{s_{i} / \phi_{i}^{2}}{\left(n \phi_{i}^{2}-B_{i} \phi_{i}^{3}\right) / \theta_{i}^{3}}
$$

can be seen to be an increasing function of $\theta_{i}$.

Now, $\frac{\partial \log L}{\partial \theta_{i}}$ is monotonically decreasing (proved in Appendix-Lemma 1) in $\theta_{i}$ so that $\frac{\partial \log L}{\partial \theta_{i}}$ has same sign for all values of $\theta_{i}$ between $\theta_{i}^{(t)}$ and $\theta_{i}^{(t+1)}$. Thus the change in the likelihood $\Delta \log L=\left.\Delta \theta_{i} \frac{\partial \log L}{\partial \theta_{i}}\right|_{\epsilon} \geq 0$ with equality iff $\Delta \theta_{i}=0$, where, $\left.\frac{\partial \log L}{\partial \theta_{i}}\right|_{\epsilon}$ denotes $\frac{\partial \log L}{\partial \theta_{i}}$ at $\theta_{i}^{(n)}+\epsilon J_{i} \Delta \theta_{i}$ for $0<\epsilon<1$ and $J_{i}$ being the vector whose $i$ th element is 1 and all the other elements are equal to 0 . Therefore, it is proved that likelihood is increased at every step of the iterative scheme if and only if the corresponding parameter value is changed.

Case II. $B_{i}>0$.

In this case, it is shown that $\frac{\theta_{i}^{3} B_{i}}{\phi_{i}^{3}}$ is an increasing function of $\theta_{i}$ (proved in Appendix-Lemma 3). Now,

$$
\begin{aligned}
\left.\frac{\partial \log L}{\partial \theta_{i}}\right|_{t} & =\left.\frac{\phi_{i}^{3}}{\theta_{i}^{4}}\left[\frac{\theta_{i}^{3} s_{i}}{\phi_{i}^{4}}-\frac{n \theta_{i}^{4}}{\phi_{i}^{4}}+\frac{B_{i} \theta_{i}^{4}}{\phi_{i}^{3}}\right]\right|_{t} \\
& =\frac{n \phi_{i}^{3}}{\theta_{i}^{4}}\left[\frac{1}{n}\left(\frac{\theta_{i}^{3} s_{i}}{\phi_{i}^{4}}+\frac{B_{i} \theta_{i}^{4}}{\phi_{i}^{3}}\right)^{(t)}-\left(\frac{\theta_{i}^{4}}{\phi_{i}^{4}}\right)^{(t)}\right] \\
& =\frac{n \phi_{i}^{3}}{\theta_{i}^{4}}\left[\left(\frac{\theta_{i}^{4}}{\phi_{i}^{4}}\right)^{(t+1)}-\left(\frac{\theta_{i}^{4}}{\phi_{i}^{4}}\right)^{(t)}\right], \text { from }(2.10),
\end{aligned}
$$


which has the same sign as that of $\Delta \theta_{i}=\theta_{i}^{(t+1)}-\theta_{i}^{(t)}$ since $\theta_{i} / \phi_{i}$ is an increasing function of $\theta_{i}$. Next,

$$
\begin{aligned}
\left.\frac{\partial \log L}{\partial \theta_{i}}\right|_{t+1} & =\frac{\phi_{i}^{3}}{\theta_{i}^{4}}\left[\left(\frac{\theta_{i}^{3} s_{i}}{\phi_{i}^{4}}+\frac{B_{i} \theta_{i}^{4}}{\phi_{i}^{3}}\right)^{(t+1)}-n\left(\frac{\theta_{i}^{4}}{\phi_{i}^{4}}\right)^{(t+1)}\right] \\
& =\frac{\phi_{i}^{3}}{\theta_{i}^{4}}\left[\left(\frac{\theta_{i}^{3} s_{i}}{\phi_{i}^{4}}+\frac{B_{i} \theta_{i}^{4}}{\phi_{i}^{3}}\right)^{(t+1)}-\left(\frac{\theta_{i}^{3} s_{i}}{\phi_{i}^{4}}+\frac{B_{i} \theta_{i}^{4}}{\phi_{i}^{3}}\right)^{(t)}\right],
\end{aligned}
$$

which has the same sign as $\Delta \theta_{i}$ since $\frac{\theta_{i}^{3} s_{i}}{\phi_{i}^{4}}+\frac{B_{i} \theta_{i}^{4}}{\phi_{i}^{3}}$ is an increasing function of $\theta_{i}$. Again, since $\theta_{i} \frac{\partial \log L}{\partial \theta_{i}}$ is monotone decreasing in $\theta_{i}$ so the result follows as in the case I.

\section{$3 \quad$ Testing Order Effects}

It is important to test whether the probability of choosing object $O_{i}$ in the ordered pair $\left(O_{i}, O_{j}\right)$ is same as the probability of choosing $O_{i}$ in the ordered pair $\left(O_{j}, O_{i}\right)$ for all $\left(O_{i}, O_{j}\right)$ or not. Thus, the null hypothesis of interest could be formulated as follows.

$$
H_{0}^{1}: \theta_{i j}=1-\theta_{j i} \forall i, j ; i \neq j,
$$

(or, equivalently $\theta_{i}=1-\theta_{K+i}, i=1,2, \cdots, K$, where, $K=m(m-1) / 2$ ).

Under $H_{0}^{1}, p(\mathbf{y})$ in $(2.5)$ reduces to

$$
p_{0}(\mathbf{y})=p_{01}(\mathbf{y}) h_{0}(\mathbf{y})
$$

where,

$$
p_{01}(\mathbf{y})=\prod_{i=1}^{K} \theta_{i}^{\left(1+y_{i}-y_{K}+i\right)} \phi_{i}^{\left(1-y_{i}+y_{K}+i\right)} .
$$

Under $H_{0}^{1}$, the log-likelihood function $L_{01}(\mathbf{y})$ is then given by

$$
\sum_{i=1}^{K}\left(n+s_{i}-s_{K+i}\right) \log \theta_{i}+\left(n-s_{i}+s_{K+i}\right) \log \left(1-\theta_{i}\right)+\sum_{y} n(\mathbf{y}) \log h_{0}^{1}(\mathbf{y}),
$$

where,

$$
\begin{aligned}
h_{0}^{1}(\mathbf{y})= & 1+\sum_{i<j} u(i, j) \delta\left(y_{i}, y_{j}\right) \psi_{i}^{\delta\left(y_{i}, 1\right) / 2} \psi_{j}^{\delta\left(y_{j}, 1\right) / 2} \\
\psi_{i} & = \begin{cases}\phi_{i} / \theta_{i} & \text { if } i=1,2, \cdots, K, \\
\theta_{j} / \phi_{j} & \text { if } i=K+1, K+2, \cdots, M ; j=i-K .\end{cases}
\end{aligned}
$$

The maximum likelihood estimates $r_{i j}$ of $\rho_{i j} ; i<j ; i, j=1,2, \cdots, M$ and $q_{i}$ of $\theta_{i} ; i=1,2, \cdots, K$, under the null hypothesis $H_{0}^{1}$ are obtained by solving the following equations.

$$
\begin{gathered}
\sum_{y} \frac{n(\mathbf{y})}{h_{0}^{1}(\mathbf{y})} \frac{\partial h_{0}^{1}(\mathbf{y})}{\partial r_{i j}}=0 ; i, j=1,2, \cdots, M, i<j, \\
\frac{n+s_{i}-s_{K+i}}{q_{i}}-\frac{n-s_{i}+s_{K+i}}{p_{i}}+\sum_{y} \frac{n(\mathbf{y})}{h_{0}^{1}(\mathbf{y})} \frac{\partial h_{0}^{1}(\mathbf{y})}{\partial q_{i}}=0 ; i=1,2, \cdots, K,
\end{gathered}
$$


where,

$$
\begin{aligned}
\frac{\partial h_{0}^{1}(\mathbf{y})}{\partial r_{i j}}= & u(i, j) \delta\left(y_{i}, y_{j}\right) \psi_{i}^{\delta\left(y_{i}, 1\right) / 2} p s i_{j}^{\delta\left(y_{j}, 1\right) / 2}, \\
\frac{\partial h_{0}^{1}(\mathbf{y})}{\partial q_{i}}= & -\frac{1}{2 p_{i} q_{i}}\left[\left(\frac{p_{i}}{q_{i}}\right)^{\delta\left(y_{i}, 1\right) / 2} \sum_{j, k \neq i} u(i, j) r_{i j} \delta\left(y_{j}, 1\right)\left(\frac{p_{j}}{q_{j}}\right)^{\delta\left(y_{i}, 1\right) / 2}\right. \\
& -r_{i, k+1}\left[\delta\left(y_{i}, 1\right)-\delta\left(y_{k+i}, 1\right)\right]\left(\frac{p_{i}}{q_{i}}\right)^{\left(\delta\left(y_{i}, 1\right)-\delta\left(y_{k+i}, 1\right)\right) / 2} \\
& \left.-\left(\frac{p_{i}}{q_{i}}\right)^{\delta\left(y_{k+i}, 1\right) / 2} \sum_{j \neq i} u(k+i, j) r_{k+i, j} \delta\left(y_{j}, 1\right)\left(\frac{p_{j}}{q_{j}}\right)^{\delta\left(y_{j}, 1\right) / 2}\right] .
\end{aligned}
$$

Using the similar iterative schemes and similar arguments to show convergence of the iterative schemes to the solutions of (3.1) and (3.2) we get the maximum likelihood estimates of the parameters under the null hypothesis $H_{0}^{1}$.

Let $r$ and $q$ be the maximum likelihood estimates under model and $r^{(01)}$ and $q^{(01)}$ be maximum likelihood estimates of $\rho$ and $\theta$ under $H_{0}^{1}$. Then, the likelihood ratio criterion is given by:

$$
\lambda_{1}=\frac{L_{01}\left(y \mid r^{(01)}, q^{(01)}\right)}{L(y \mid r, q)} .
$$

The hypothesis $H_{0}^{1}$ is rejected if $\lambda_{1}$ in (3.3) is small or equivalently, $-2 \log \lambda_{1}$ is large. Under $H_{0}^{1},-2 \log \lambda_{1}$ is distributed asymptotically as $\chi^{2}$ with $K$ df.

\section{Correlation of Judgments on Different Pairs}

One may be interested in knowing whether the judgment on the pair $\left(O_{i_{1}}, O_{j_{1}}\right)$ is uncorrelated with the judgment on the pair $\left(O_{k_{1}}, O_{l_{1}}\right)$. We can test the hypothesis:

$$
H_{0}^{2}: \rho_{\left(i_{1}, j_{1}\right),\left(k_{1}, l_{1}\right)}=0 \quad \text { for some }\left(i_{1}, j_{1}\right),\left(k_{1}, l_{1}\right) .
$$

Under the null hypothesis $H_{0}^{2}, \log L$ in (2.6) reduces to:

$$
\log L_{02}=\sum_{i=1}^{M}\left[s_{i} \log \theta_{i}+\left(n-s_{i}\right) \log \left(1-\theta_{i}\right)\right]+\sum_{y} n(\mathbf{y}) \log h_{0}^{2}(\mathbf{y})
$$

where,

$$
h_{0}^{2}(\mathbf{y})=1+\sum_{i<j}^{*} u(i, j) \rho_{i j} Z_{i} Z_{j},
$$

$\sum^{*}$ denoting the summation over other indices except the particular $\left(i_{1}, j_{1}\right),\left(k_{1}, l_{1}\right)$. We get similar sets of likelihood equations. Using the same type of iterative schemes, we find the maximum likelihood estimates of parameters under hypothesis $H_{0}^{2}$.

Let $r^{(02)}$ and $q^{(02)}$ be maximum likelihood estimates of $\rho$ and $\theta$ under $H_{0}^{2}$. Then, the likelihood ratio criterion is given by:

$$
\lambda_{2}=\frac{L_{02}\left(y \mid r^{(02)}, q^{(02)}\right)}{L(y \mid r, q)} .
$$


The hypothesis $H_{0}^{2}$ is rejected if $\lambda_{2}$ in (4.1) is small or equivalently, $-2 \log \lambda_{2}$ is large. Under $H_{0}^{2},-2 \log \lambda_{2}$ is distributed asymptotically as $\chi^{2}$ with $1 \mathrm{df}$.

Sometimes, we may think that the correlations of certain number of pairs of treatments are same. More specifically, we may suspect that correlation between the pair $\left(O_{i_{1}}, O_{j_{1}}\right)$ and the pair $\left(O_{i_{2}}, O_{j_{2}}\right)$ is same as the correlation between $\left(O_{i_{1}}, O_{j_{1}}\right)$ and the pair $\left(O_{i_{3}}, O_{j_{3}}\right)$ and so on up to the correlation between $\left(O_{i_{1}}, O_{j_{1}}\right)$ and $\left(O_{i_{k}}, O_{j_{k}}\right)$. We may test the following null hypothesis:

$$
H_{0}^{3}: \rho_{\left(i_{1}, j_{1}\right),\left(i_{2}, j_{2}\right)}=\rho_{\left(i_{1}, j_{1}\right),\left(i_{3}, j_{3}\right)}=\cdots=\rho_{\left(i_{1}, j_{1}\right),\left(i_{k}, j_{k}\right)}=\rho_{0}, \text { say }
$$

The $\log L$ in (2.6), under the hypothesis $H_{0}^{3}$, reduces to:

$$
\log L_{03}=\sum_{i=1}^{M}\left[s_{i} \log \theta_{i}+\left(n-s_{i}\right) \log \left(1-\theta_{i}\right)\right]+\sum_{y} n(\mathbf{y}) \log h_{0}^{3}(\mathbf{y}),
$$

where,

$$
h_{0}^{3}(\mathbf{y})=1+\sum_{i<j}^{* *} u(i, j) \rho_{i j} Z_{i} Z_{j}+\rho_{0} \sum_{l=1}^{k} u\left(i_{1}, j_{1}\right) Z_{i_{l}} Z_{j_{l}},
$$

$\sum^{* *}$ denoting the summation over other indices except the $\left(i_{1}, j_{1}\right),\left(i_{2}, j_{2}\right) ;\left(i_{1}, j_{1}\right),\left(i_{3}, j_{3}\right) ; \cdots ;\left(i_{1}, j_{1}\right)$, $\left(i_{k}, j_{k}\right)$. Then the maximum likelihood estimates of $\rho_{0}$ and $\theta_{i}$ s respectively are obtained from the following sets of equations:

$$
\begin{aligned}
\sum_{y} \frac{n(\mathbf{y})}{h_{0}^{3}(\mathbf{y})} \frac{\partial h_{0}^{3}(\mathbf{y})}{\partial \rho_{0}} & =0, \\
\frac{s_{i}-n q_{i}}{q_{i} p_{i}}-\sum_{y} \frac{n(\mathbf{y})}{h_{0}^{3}(\mathbf{y})} \frac{\partial h_{0}^{3}(\mathbf{y})}{\partial q_{i}} & =0 ; i=1,2, \cdots, M, \\
\frac{1}{2 q_{i} p_{i}}\left(\frac{q_{i}}{p_{i}}\right)^{\delta\left(y_{i}, 1\right) / 2} u(i, j) \delta\left(y_{j}, 1\right)\left(\frac{q_{j}}{p_{j}}\right)^{\delta\left(y_{j}, 1\right)} & =m_{i j}, \quad p_{i}=1-q_{i},
\end{aligned}
$$

where,

$$
\begin{gathered}
\frac{\partial h_{0}^{3}(\mathbf{y})}{\partial \rho_{0}}=\sum_{l=1}^{k} u\left(i_{l}, j_{l}\right) Z_{i_{l}} Z_{j_{l}}, \\
\frac{\partial h_{0}^{3}(\mathbf{y})}{\partial \theta_{i}}=\sum_{j \neq i} m_{i j} r_{i j}^{*}, \\
r_{i j}^{*}= \begin{cases}r_{i j} & \text { if }(i, j) \neq\left(i_{1}, j_{1}\right),\left(i_{2}, j_{2}\right) ;\left(i_{1}, j_{1}\right),\left(i_{3}, j_{3}\right) ;\left(i_{1}, j_{1}\right),\left(i_{k}, j_{k}\right), \\
\rho_{0} & \text { otherwise. }\end{cases}
\end{gathered}
$$

along with (3.2) for other estimates $r_{i j} \mathrm{~s}$ of $\rho_{i j} \mathrm{~s}$.

Let $r^{(03)}$ and $q^{(03)}$ be maximum likelihood estimates of $\rho$ and $\theta$ under $H_{0}^{3}$. Then, the likelihood ratio criterion is given by:

$$
\lambda_{3}=\frac{L_{(03)}\left(\mathbf{y} \mid \mathbf{r}^{(03)}, \mathbf{q}^{(03)}\right)}{L(\mathbf{y} \mid \mathbf{r}, \mathbf{q})} .
$$

The hypothesis $H_{0}^{3}$ is rejected if $\lambda_{3}$ in (4.2) is small or equivalently, $-2 \log \lambda_{3}$ is large. Under $H_{0}^{3},-2 \log \lambda_{3}$ is distributed asymptotically as $\chi^{2}$ with $k-1 \mathrm{df}$. 


\section{$5 \quad$ Estimation and Test for Equality of Priority Vectors}

The estimates of priority vector $\boldsymbol{\pi}=\left(\pi_{1}, \pi_{2}, \cdots, \pi_{m}\right)^{\prime}$ of the alternatives may be found as follows. Define,

$$
\begin{aligned}
\mu_{i} & =\sum_{j \neq i} \frac{\theta_{i j}+1-\theta_{j i}}{2}, \\
\pi_{i} & =\frac{\mu_{i}}{\sum_{j=1}^{m} \mu_{j}} ; i=1,2, \cdots, m .
\end{aligned}
$$

The maximum likelihood estimates $m_{i}$ of $\mu_{i}$ and $p_{i}$ of $\pi_{i}$ are given (with $q_{i j}$ s as estimates of $\theta_{i j s} \mathrm{~s}$ ) respectively by:

$$
\begin{aligned}
m_{i} & =\sum_{j \neq i} \frac{q_{i j}+1-q_{j i}}{2}, \\
p_{i} & =\frac{m_{i}}{\sum_{j=1}^{m} m_{j}}
\end{aligned}
$$

The hypothesis of equal priority of the alternatives is:

$$
H_{0}^{4}: \pi_{1}=\pi_{2}=\cdots=\pi_{m} .
$$

Under $H_{0}^{4}$, the number of parameters $\theta_{i j} \mathrm{~s}$ is reduced by $m-1$. Under $H_{0}^{4}$, estimates $q_{i j} \mathrm{~s}$ of $\theta_{i j}$ s are given by:

$$
q_{1 i}=q_{i 1}+\frac{\sum_{j \neq 1, i}\left(q_{i j}+1-q_{j i}\right)-\sum_{j \neq 1, i}\left(q_{1 j}+1-q_{j 1}\right)}{2} ; i=1,2, \cdots, m .
$$

The estimates of the other $\theta_{i j}$ s are obtained by (2.7) and (2.8). The hypothesis $H_{0}^{4}$ is rejected if $-2 \log \lambda_{4}$ is large where, $\lambda_{4}$ is the corresponding likelihood ratio statistic. Under $H_{0}^{4},-2 \log \lambda_{4}$ is distributed asymptotically as $\chi^{2}$ with $m-1 \mathrm{df}$.

\section{Discussion}

In this paper we have generalized the idea of dependence of judgments for Analytic Hierarchy Process.

\section{References}

[1] Bahadur, R. R. (1961). A representation of the joint distribution of responses to $\mathrm{n}$ dichotomous items. In Studies in item Analysis and Prediction, Ed. H. Solomon, 158-168. Stanford University Press.

[2] Beaver, R. J. and Gokhale, D. D. (1975). A model to incorporate within -pair order effects in paired comparisons. Communications in Statistics 4, 923-939.

[3] Davidson, R. R. (1970). On extending the Bradley-Terry model to accomodate ties in paired comparison experiments. Journal of The American Statistical Association 65, 317-328.

[4] Davidson, R. R. and Beaver, R. J. (1977). On extending the Bradley-Terry model to incorporate within-pair order effects. Biometrics 33, 24 5-254. 
[5] Davidson, R. R. and Bradley, R. A. (1969). Multivariate paired comparisons: The extension of a univariate model and associated estimation and test procedures. Biometrika 56, 81-95.

[6] Davidson, R. R. and Bradley, R. A. (1971). A regression relationship for multivariate paired comparisons. Biometrika 58, 555-560.

[7] De Jong, P. (1984). A statistical approach to Saaty's scaling method for priorities. Journal of Mathematical Psychology 28, 467-478. 


\section{Appendix}

Lemma 1. $\theta_{i} \frac{\partial \log L}{\partial \theta_{i}}$ is monotone decreasing in $\theta_{i}$ except for very small values of $\theta_{i}$.

Proof. We will show that $\frac{\partial \log L}{\partial \theta_{i}}+\theta_{i} \frac{\partial^{2} \log L}{\partial \theta_{i}^{2}}$ is negative.

$$
\frac{\partial \log L}{\partial \theta_{i}}=\frac{s_{i}}{\theta_{i}}-\frac{n-s_{i}}{1-\theta_{i}}+\sum_{y} \frac{n(\mathbf{y}) h^{\prime}(\mathbf{y})}{h(\mathbf{y})}
$$

and

$$
\frac{\partial^{2} \log L}{\partial \theta_{i}^{2}}=-\frac{s_{i}}{\theta_{i}^{2}}-\frac{n-s_{i}}{\left(1-\theta_{i}\right)^{2}}+\sum_{y} \frac{n(\mathbf{y}) h^{\prime \prime}(\mathbf{y})}{h(\mathbf{y})}-\sum_{y} \frac{n(\mathbf{y})\left\{h^{\prime}(\mathbf{y})\right\}^{2}}{\{h(\mathbf{y})\}^{2}}
$$

where,

$$
\begin{aligned}
h^{\prime}(\mathbf{y}) & =-\frac{1}{2 \phi_{i} \theta_{i}}\left(\frac{\phi_{i}}{\theta_{i}}\right)^{\delta\left(y_{i}, 1\right) / 2} C_{i}, \\
h^{\prime \prime}(\mathbf{y}) & =\frac{\delta\left(y_{i}, 1\right)+2-4 \theta_{i}}{4 \theta_{i}^{2} \phi_{i}^{2}}\left(\frac{\phi_{i}}{\theta_{i}}\right)^{\delta\left(y_{i}, 1\right) / 2} C_{i}, \\
C_{i} & =\sum_{j \neq i} u(i, j) \delta\left(y_{j}, 1\right) \rho_{i j}\left(\frac{\phi_{j}}{\theta_{j}}\right)^{\delta\left(y_{j}, 1\right) / 2} .
\end{aligned}
$$

Then

$$
\begin{aligned}
\frac{\partial \log L}{\partial \theta_{i}}+\frac{\partial^{2} \log L}{\partial \theta_{i}^{2}} & =\sum \frac{n(\mathbf{y})}{h(\mathbf{y})} \frac{C_{i}}{4 \theta_{i} \phi_{i}^{2}}\left(\frac{\phi_{i}}{\theta_{i}}\right)^{\delta\left(y_{i}, 1\right) / 2} \\
& {\left[\delta\left(y_{i}, 1\right)-2 \theta_{i} \frac{C_{i}}{h(\mathbf{y})}\left(\frac{\phi_{i}}{\theta_{i}}\right)^{\delta\left(y_{i}, 1\right) / 2}-\frac{h(\mathbf{y})}{C_{i}}\left(\frac{\theta_{i}}{\phi_{i}}\right) 4 \theta_{i}\left(1-\frac{s_{i}}{n}\right)\right], }
\end{aligned}
$$

which is seen to be negative in all the cases. Hence it is proved that

$$
\frac{\partial \log L}{\partial \theta_{i}}+\frac{\partial^{2} \log L}{\partial \theta_{i}^{2}}<0
$$

Lemma 2. $\frac{\phi_{i}^{3}}{\theta_{i}^{3}} \sum_{y} \frac{n(\mathbf{y}) h^{\prime}(\mathbf{y})}{h(\mathbf{y})}$ is an increasing function of $\theta_{i}$ if $B_{i}$ is negative.

Proof. $\frac{\phi_{i}^{3}}{\theta_{i}^{3}} \sum_{y} \frac{n(\mathbf{y}) h^{\prime}(\mathbf{y})}{h(\mathbf{y})}=\sum n(\mathbf{y})\left[\frac{\phi_{i}}{\theta_{i} h(\mathbf{y})}\right]\left[\frac{\phi_{i}^{2} h^{\prime}(\mathbf{y})}{\theta_{i}^{2}}\right]$

$$
\begin{gathered}
\frac{\phi_{i}}{\theta_{i} h(\mathbf{y})}=\left[\frac{\theta_{i}}{\phi_{i}}+\sum_{i<j} \delta\left(y_{i}, y_{j}\right) \rho_{i j}\left(\frac{\phi_{i}}{\theta_{i}}\right)^{\delta\left(y_{j}, 1\right) / 2}\right]^{-1} \\
\frac{\phi_{i}^{2} h^{\prime}(\mathbf{y})}{\theta_{i}^{2}}=\frac{1}{2 \phi_{i} \theta_{i}}+\left(\frac{\phi_{i}}{\theta_{i}}\right)^{2+\delta\left(y_{j}, 1\right) / 2} C_{i} .
\end{gathered}
$$

The derivative of $\frac{\phi_{i}^{3}}{\theta_{i}^{3}} \sum_{y} \frac{n(\mathbf{y}) h^{\prime}(\mathbf{y})}{h(\mathbf{y})}$ is given by: 


$$
\begin{gathered}
\sum n(\mathbf{y})\left[\frac{\phi_{i}}{\theta_{i} h(\mathbf{y})}\right]\left[\frac{\delta\left(y_{i}, 1\right) / 2+3-2 \theta_{i}}{2 \theta_{i}^{2} \phi_{i}^{2}}\right]\left(\frac{\phi_{i}}{\theta_{i}}\right)^{\delta\left(y_{i}, 1\right) / 2} C_{i} \\
-\sum n(\mathbf{y}) \frac{\phi_{i}^{3}}{\theta_{i}^{3}}\left[\frac{h^{\prime}(\mathbf{y})}{h(\mathbf{y})}\right]^{2}+\sum n(\mathbf{y}) \frac{\phi_{i}^{2}}{\theta_{i}^{4}} \frac{h^{\prime}(\mathbf{y})}{h(\mathbf{y})} .
\end{gathered}
$$

Now, $\delta\left(y_{i}, 1\right) / 2+3-2 \theta_{i}$ is always positive indicating that the first term in the above expression is positive. Combining the second and the third terms we then get,

$$
\sum n(\mathbf{y}) \frac{\phi_{i}^{2}}{\theta_{i}^{4}} \frac{h^{\prime}(\mathbf{y})}{h(\mathbf{y})}\left[1-\theta_{i} \phi_{i} \frac{h^{\prime}(\mathbf{y})}{h^{\prime \prime}(\mathbf{y})}\right]
$$

which is always positive since $\theta_{i} \phi_{i} \frac{h^{\prime}(\mathbf{y})}{h(\mathbf{y})}<1$.

Hence, the sign of (6.1) is always positive. This proves the Lemma 2.

Lemma 3. $\frac{\theta_{i}^{3}}{\phi_{i}^{3}} \sum_{y} \frac{n(\mathbf{y}) h^{\prime}(\mathbf{y})}{h(\mathbf{y})}$ is an increasing function of $\theta_{i}$ if $B_{i}$ is positive.

Proof. $\frac{\theta_{i}^{3}}{\phi_{i}^{3}} \sum_{y} \frac{n(\mathbf{y}) h^{\prime}(\mathbf{y})}{h(\mathbf{y})}=\sum n(\mathbf{y})\left[\frac{\theta_{i}}{\phi_{i} h(\mathbf{y})}\right]\left[\frac{\theta_{i}^{2} h^{\prime}(\mathbf{y})}{\phi_{i}^{2}}\right]$

$$
\begin{gathered}
\frac{\theta_{i}}{\phi_{i} h(\mathbf{y})}=\left[\frac{\phi_{i}}{\theta_{i}}+\sum_{i<j} \delta\left(y_{i}, y_{j}\right) \rho_{i j}\left(\frac{\phi_{i}}{\theta_{i}}\right)^{1+\delta\left(y_{j}, 1\right) / 2}\right]^{-1} \\
\frac{\theta_{i}^{2} h^{\prime}(\mathbf{y})}{\phi_{i}^{2}}=\frac{1}{2 \phi_{i} \theta_{i}}+\left(\frac{\phi_{i}}{\theta_{i}}\right)^{\delta\left(y_{j}, 1\right) / 2-2} C_{i} .
\end{gathered}
$$

The derivative of $\frac{\theta_{i}^{3}}{\phi_{i}^{3}} \sum_{y} \frac{n(\mathbf{y}) h^{\prime}(\mathbf{y})}{h(\mathbf{y})}$ is given by:

$$
\begin{gathered}
\sum n(\mathbf{y})\left[\frac{\theta_{i}}{\phi_{i} h(\mathbf{y})}\right]\left[\frac{\delta\left(y_{i}, 1\right) / 2-1-2 \theta_{i}}{2 \theta_{i}^{2} \phi_{i}^{2}}\right]\left(\frac{\phi_{i}}{\theta_{i}}\right)^{\delta\left(y_{i}, 1\right) / 2} C_{i} \\
-\sum n(\mathbf{y}) \frac{\theta_{i}^{3}}{\phi_{i}^{3}}\left[\frac{h^{\prime}(\mathbf{y})}{h(\mathbf{y})}\right]^{2}+\sum n(\mathbf{y}) \frac{\theta_{i}^{2}}{\phi_{i}^{4}} \frac{h^{\prime}(\mathbf{y})}{h(\mathbf{y})} .
\end{gathered}
$$

Now, $\delta\left(y_{i}, 1\right) / 2-1-2 \theta_{i}$ is always positive indicating that the first term in the above expression is positive. Combining the second and the third terms we then get,

$$
\sum n(\mathbf{y}) \frac{\theta_{i}^{2}}{\phi_{i}^{4}} \frac{h^{\prime}(\mathbf{y})}{h(\mathbf{y})}\left[1-\theta_{i} \phi_{i} \frac{h^{\prime}(\mathbf{y})}{h^{\prime \prime}(\mathbf{y})}\right]
$$

which is always positive since $\theta_{i} \phi_{i} \frac{h^{\prime}(\mathbf{y})}{h(\mathbf{y})}<1$.

Hence, the sign of (6.2) is always positive. This proves the Lemma 3. 